\title{
Estudo epidemiológico dos traumas oculares graves em um Hospital Universitário de São José do Rio Preto - SP
}

Epidemiological study of severe ocular trauma in a University Hospital of São gosé do Rio Preto - SP

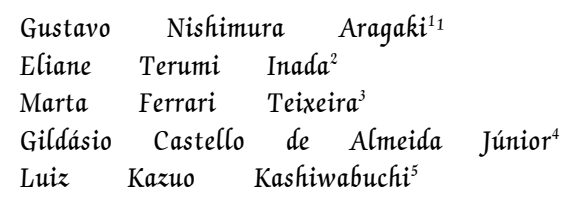

\section{RESUMO}

Objetivos: Estudar a prevalência e as circunstâncias do trauma ocular grave em um Hospital Universitário de São José do Rio Preto - SP. Métodos: Realizou-se estudo retrospectivo de 216 pacientes no período de setembro de 1986 a setembro de 2000 com traumatismo ocular grave que evoluiu para cirurgia. Foram avaliados o sexo, idade, lateralidade, causa do trauma, tipo de trauma, atividade no momento do acidente e acuidade visual póstrauma. Resultados: Foram estudados 216 pacientes sendo $173(80 \%)$ do sexo masculino. A faixa etária mais acometida foi a menor de 20 anos em $94(43,5 \%)$ pacientes. As lesões unilaterais predominaram em 209(96,8\%). A atividade predominante no momento do trauma foi o lazer em $88(40,7 \%)$ pacientes e os principais agentes causais foram os materiais de construção em $82(38 \%)$ dos casos. A perfuração ocular foi o tipo de trauma mais comum em $84(85,2 \%)$ pacientes. Conclusões: As perfurações oculares unilaterais por materiais de construção predominaram em crianças, adolescentes e idosos do sexo masculino em atividades de lazer.

Descritores: Traumatismos oculares/etiologia; Acidentes ocupacionais; Materiais de construção; Cegueira/prevenção \& controle; Saúde ocular

Trabalho realizado na disciplina de Oftalmologia do Hospital de Base da Faculdade de Medicina de São José do Rio Preto - SP - FAMERP.

${ }^{1}$ Graduando do $6^{\circ}$ ano da Faculdade de Medicina de São José do Rio Preto - FAMERP.

${ }^{2}$ Residente de Oftalmologia da Faculdade de Medicina de São José do Rio Preto.

${ }^{3}$ Professor Assistente de Oftalmologia da Faculdade de Medicina de São José do Rio Preto; Mestre em Medicina Interna.

${ }^{4}$ Médico Contratado pelo Hospital de Base - FUNFARME. Pós-graduando em Oftalmologia pela Universidade de São Paulo - Ribeirão Preto.

${ }_{5}^{5}$ Professor Adjunto de Oftalmologia da Faculdade de Medicina de São José do Rio Preto; Doutor em Oftalmologia pela Universidade Federal de Minas Gerais.

Endereço para correspondência: Dra. Eliane Terumi Inada. Rua Atílio Luiz Fasanelli n ${ }^{0} 199$ - Ap 22

São José do Rio Preto (SP) CEP 15091-300

E-mail: t inada@eudoramail.com

Recebido para publicação em 25.04.2002

Aceito para publicação em 30.10.2002

Nota Editorial: Pela análise deste trabalho e por sua anuência na divulgação desta nota, agradecemos à Dra. Denise de Freitas.

\section{INTRODUÇÃO}

Os traumatismos oculares são responsáveis por significativa procura aos serviços de Oftalmologia, pois causam alteração ocular funcional substancial podendo até levar à cegueira, com prejuízos pessoais, sociais e econômicos importantes ${ }^{(1)}$.

Os acidentes oculares variam desde pequenas lesões do tipo abrasões até perfurações extensas graves, necessitando de uma urgente intervenção oftalmológica.

A adoção da prevenção na área de Oftalmologia tem crescido em importância, ampliando a função do médico oftalmologista de apenas diagnosticar e tratar doenças ${ }^{(2)}$. Desta forma, torna-se necessário o conhecimento da epidemiologia do trauma ocular responsável pela diminuição da capacidade visual por fatores facilmente preveníveis.

Este trabalho tem como objetivo levantar a prevalência e as circunstâncias do trauma ocular grave dos pacientes atendidos no Serviço de Oftalmologia do Hospital de Base da Faculdade de Medicina de São José do Rio Preto - SP. 


\section{MÉTODOS}

Este trabalho avaliou retrospectivamente 216 pacientes admitidos no Serviço de Oftalmologia do Hospital de Base da Faculdade de Medicina de São José do Rio Preto - SP no período de Setembro de 1986 a Setembro de 2000. Para tal avaliação, utilizou-se um protocolo baseado no prontuário médico, considerando alguns critérios de inclusão e exclusão. Os critérios de inclusão analisados foram: 1) diagnóstico de trauma ocular grave (considerou-se como grave apenas os traumas oculares que tiveram indicação cirúrgica); 2) sexo e idade; 3) profissão; 4) lateralidade; 5) atividade no momento do acidente (domiciliar, lazer, trabalho, trânsito e violência); 6) causa do trauma (acidente automobilístico, arma branca, arma de fogo, fogos de artifício, galho de árvore, materiais domésticos, materiais de construção, materiais infanto-escolares e outros); 7) tipo de trauma (ferimento contuso, ferimento perfurante, ferimento perfurante e corpo estranho intra-ocular, queimadura química e semi-avulsão do globo ocular); 8) acuidade visual final.

O único critério de exclusão foi a presença de dados incompletos no prontuário médico para preenchimento do protocolo.

A avaliação da acuidade visual foi baseada na tabela de Snellen.

Os pacientes foram divididos, para efeito de estudo da faixa etária, em sete grupos: até 10 anos, de 11 a 20 anos, de 21 a 30 anos, de 31 a 40 anos, de 41 a 50 anos, de 51 a 60 anos e maior de 60 anos.

\section{RESULTADOS}

O resultado da análise dos 216 pacientes mostrou que 173 (80\%) eram do sexo masculino e $43(20 \%)$, do sexo feminino. Considerando-se a faixa etária, $48(22,2 \%)$ pacientes encontram-se na faixa de 0 a 10 anos; $46(21,3 \%)$ entre 11 a 20 anos; $40(18,5 \%)$ entre 21 a 30 anos; $31(14,4 \%)$ entre 31 a 40 anos; $21(9,7 \%)$ entre 41 a 50 anos; $13(6 \%)$ entre 51 a 60 anos e 17 (7,9\%) com mais de 60 anos. A idade dos pacientes variou de 1 a 89 anos, sendo a média de $28,3 \pm 20,5$ anos. De acordo com a lateralidade, o olho esquerdo foi acometido em 111 $(51,4 \%)$ pacientes, o olho direito em $98(45,4 \%)$ e ambos os olhos em $7(3,2 \%)$.
Quanto à atividade no momento do trauma, verificou-se que $88(40,7 \%)$ pacientes encontravam-se em atividades de lazer; 62 (28,7\%) no trabalho; 23 (10,6\%) no trânsito; 21 (9,7\%) em ambiente doméstico e 21 (9,7\%) em decorrência de violência. $\mathrm{O}$ resultado dos traumas oculares em relação à atividade no momento do trauma e faixa etária está disposto na tabela 1 .

Os agentes causadores do trauma ocular foram variados, predominando as lesões por materiais de construção em 82 (38\%) pacientes, seguido por galho de árvore em 33 (15,3\%) pacientes e acidente automobilístico em 23 (10,6\%) pacientes. Outros agentes traumáticos também foram responsáveis pelas lesões oculares (Tabela 2).

A distribuição dos traumas oculares graves em relação ao agente causador e a atividade no momento do trauma encontram-se na tabela 3 .

Dentre os tipos de trauma ocorridos, os mais freqüentes foram a perfuração ocular em $84(85,2 \%)$ casos (Tabela 4$)$.

Correlacionando os fatores acima analisados, temos que os ferimentos oculares ocorridos durante atividades de lazer, predominaram no sexo masculino em 65 (74\%) pacientes e atingiram indivíduos menores de 20 anos em 64 (73\%) casos. As perfurações oculares totalizaram $82(93,2 \%)$ dos casos sendo em sua maioria unilaterais $85(96,6 \%)$ e causados principalmente por materiais de construção 31 (35,3\%) seguidos pela madeira $20(22,6 \%)$ e pela pedra em $20(22,6 \%)$. A tabela 5 mostra a distribuição dos pacientes quanto à acuidade visual final no lazer.

O procedimento cirúrgico mais realizado nos traumas decorridos do lazer foi a sutura córneo-escleral em 46 (52,2\%) casos seguidos de evisceração do globo ocular em 24 (27,3\%), facectomia extracapsular com implante de lente intra-ocular em $4(16 \%)$ e ceratoplastia penetrante em $4(4,5 \%)$.

O perfil dos indivíduos vítimas de acidente de trabalho foi predominante no sexo masculino em $98,3 \%$ dos casos, na faixa etária entre 21 a 60 anos em $92 \%$. As profissões mais acometidas foram as de lavrador em $41(66,2 \%)$ casos, pedreiro em 12 $(19,4 \%)$ e mecânico em $3(4,8 \%)$ casos. Os traumas decorrentes do trabalho acarretaram ferimentos perfurantes em $56(90,3 \%)$ casos, na sua grande maioria unilaterais em 61 (98\%) e com visão prejudicada de 20/200 a percepção luminosa e sem percepção luminosa em 29 (46,7\%) casos.

\begin{tabular}{|c|c|c|c|c|c|c|c|}
\hline \multirow{2}{*}{$\begin{array}{l}\text { Atividade no } \\
\text { momento do } \\
\text { trauma }\end{array}$} & \multicolumn{7}{|c|}{ Faixa etária } \\
\hline & $\begin{array}{c}<11 \text { anos } \\
\%\end{array}$ & $\begin{array}{c}11 \text { a } 20 \text { anos } \\
\%\end{array}$ & $\begin{array}{c}21 \text { a } 30 \text { anos } \\
\%\end{array}$ & $\begin{array}{c}31 \text { a } 40 \text { anos } \\
\%\end{array}$ & $\begin{array}{c}41 \text { a } 50 \text { anos } \\
\%\end{array}$ & $\begin{array}{c}51 \text { a } 60 \text { anos } \\
\%\end{array}$ & $\begin{array}{c}>60 \text { anos } \\
\%\end{array}$ \\
\hline Lazer & 87,5 & 52,2 & 17,5 & 16,0 & 0 & 30,7 & 41,2 \\
\hline Trabalho & 0 & 15,2 & 42,5 & 48,4 & 57,1 & 46,2 & 29,4 \\
\hline Trânsito & 2,1 & 15,2 & 12,5 & 19,4 & 14,3 & 7,7 & 0 \\
\hline Domiciliar & 8,3 & 6,5 & 7,5 & 6,5 & 9,5 & 15,4 & 29,4 \\
\hline Violência & 2,1 & 10,9 & 20,0 & 9,7 & 19,1 & 0 & 0 \\
\hline
\end{tabular}




\begin{tabular}{|lcc|}
\hline Tabela 2. Trauma ocular - distribuição dos casos quanto ao agente \\
causador \\
Causa do trauma & $\mathbf{n}$ & $\%$ \\
Materiais de construção & 82 & 38,0 \\
Galho de árvore & 33 & 15,3 \\
Acidente automobilístico & 23 & 10,6 \\
Arma branca & 18 & 8,3 \\
Materiais domésticos & 18 & 8,3 \\
Materiais infanto-escolares & 15 & 7,0 \\
Arma de fogo & 10 & 4,6 \\
Fogos de artifício & 8 & 3,7 \\
Outros & 9 & 4,2 \\
Total & 216 & 100,0 \\
Hospital de Base de São José do Rio Preto, $1986-2000$ & \\
\hline
\end{tabular}

DISCUSSÃO

Considerando-se o sexo dos 216 pacientes estudados, o masculino foi o mais acometido por exercer atividade de maior risco em relação ao feminino, na proporção de 4:1. Resultados semelhantes foram encontrados na literatura $(3,88: 1)^{(3)} \mathrm{e}$ $(3,9: 1)^{(4)}$. Os acidentes predominaram na faixa etária de 0 a 40 anos em $76,4 \%$ dos ferimentos, atingindo crianças e jovens em idade produtiva. Isso revela a importância econômica dos traumas oculares para a sociedade.

Quanto à lateralidade, houve um predomínio das lesões unilaterais $(96,8 \%)$ sobre as bilaterais $(3,2 \%)$, sem diferenças entre o olho esquerdo em relação ao direito.

Os ferimentos ocorridos durante as atividades de lazer tiveram maior importância nesta casuística $(40,7 \%)$, seguidos pelos acidentes ocupacionais $(28,7 \%)$ e pelos acidentes automobilísticos $(10,6 \%)$. Por outro lado, alguns autores observaram apenas $4,2 \%$ de traumas oculares no lazer, mas com predominância dos acidentes automobilísticos em $29,5 \%{ }^{(5)}$ e 77,92\% de lesões oculares devido às atividades domésticas ${ }^{(6)}$. Esta variação de dados pode ser explicada pela heterogeneidade dos hábitos e costumes de cada região ou país, influenciando a causa do trauma ocular e em conseqüência, os resultados referentes à atividade no momento do acidente ${ }^{(7)}$.
Com relação a menor prevalência da violência $(9,7 \%)$ como agente causador do trauma ocular em comparação com outros trabalhos $(22,1 \%)^{(5)} \mathrm{e}(21 \%)^{(8)}$, pode ser devido ao fato de que estes foram realizados em grandes centros urbanos, locais onde se concentram os índices de violência, intensificados por piores desigualdades socioeconômicas, crescimento urbano desenfreado e o grande contingente populacional, diferenciando-se do interior, principalmente do Estado de São Paulo.

Quanto aos traumas oculares durante as atividades recreativas, $74,2 \%$ ocorreram em indivíduos menores de 21 anos, do sexo masculino, causados principalmente por arames, pedaços de madeira e galhos de árvores. Tais dados sugerem a maior atividade física e recreativa desta faixa etária. Vários autores citam a falta de atenção dos pais acerca de objetos com potencial perigo que ficam ao alcance dos filhos e que algumas medidas preventivas poderiam facilmente evitar tais prejuízos visuais $^{(9)}$.

O perfil dos indivíduos vítimas de acidente de trabalho caracterizou-se por uma média de idade de 37,3 anos, predominando a profissão lavrador. Estes resultados podem ser explicados pela forte influência da atividade agropecuária da região de São José do Rio Preto - SP. Alguns autores ressaltam a importância econômica dos traumatismos oculares graves para o sistema previdenciário e também para outros setores da economia, uma vez que a ausência do trabalhador altera negativamente a sua produção ${ }^{(10)}$. Outros referem que a alta prevalência dos acidentes oculares no trabalho não se deve à falta de leis e medidas preventivas, mas sim do não cumprimento destas pelo empregado e empregador de uma maneira geral ${ }^{(11)}$. Além disso, concluíram a necessidade por parte das empresas de desenvolver mecanismos que tornem o uso dos equipamentos mais aceitáveis e confortáveis e estimular a percepção da necessidade dos mesmos por parte dos empregados ${ }^{(11)}$.

Por fim, as lesões oculares por acidentes automobilísticos totalizaram a terceira maior causa dos traumas. Comparandose os períodos de 1990 a 1995 em relação aos períodos de 1996 a de 2000, observou-se uma diminuição dos acidentes de $43,5 \%$ para $26,1 \%$, respectivamente. Estes dados podem ser

\begin{tabular}{|c|c|c|c|c|c|}
\hline \multirow[b]{2}{*}{ Agente causador } & \multicolumn{5}{|c|}{ Atividade no momento do trauma } \\
\hline & $\begin{array}{l}\text { Lazer } \\
\%\end{array}$ & $\begin{array}{c}\text { Trabalho } \\
\%\end{array}$ & $\begin{array}{c}\text { Domiciliar } \\
\%\end{array}$ & $\begin{array}{c}\text { Violência } \\
\%\end{array}$ & $\begin{array}{c}\text { Trânsito } \\
\%\end{array}$ \\
\hline Materiais de construção & 34,8 & 64,5 & 19,0 & 33,3 & 0 \\
\hline Galho de árvore & 22,5 & 14,5 & 19,0 & 0 & 0 \\
\hline Acidente automobilístico & 0 & 0 & 0 & 0 & 100 \\
\hline Arma branca & 7,9 & 6,5 & 14,3 & 19,0 & 0 \\
\hline Materiais domésticos & 7,9 & 12,9 & 33,3 & 0 & 0 \\
\hline Materiais infanto-escolares & 13,5 & 0 & 9,6 & 4,8 & 0 \\
\hline Arma de fogo & 1,1 & 0 & 0 & 42,9 & 0 \\
\hline Fogos de artifício & 9,0 & 0 & 0 & 0 & 0 \\
\hline Outros & 3,4 & 1,6 & 4,8 & 0 & 0 \\
\hline
\end{tabular}




\begin{tabular}{|c|c|c|}
\hline Tipo de trauma & $f$ & $\%$ \\
\hline Ferimento perfurante & 184 & 85,2 \\
\hline Ferimento contuso & 22 & 10,2 \\
\hline $\begin{array}{l}\text { Ferimento perfurante e } \\
\text { corpo estranho intra-ocular }\end{array}$ & 5 & 2,3 \\
\hline Queimadura química & 4 & 1,8 \\
\hline Semi-avulsão do globo ocular & 1 & 0,5 \\
\hline Total & 216 & 100,0 \\
\hline
\end{tabular}

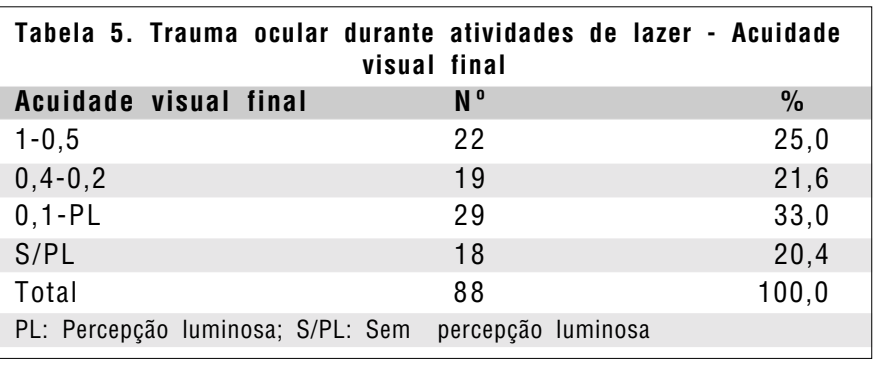

explicados pela adoção da lei que obriga o uso do cinto de segurança no Estado de São Paulo, promulgada em 1995, uma vez que o cinto de segurança evita o choque direto do rosto contra o pára-brisa do automóvel.

\section{CONCLUSÃO}

A atividade de lazer foi a principal responsável pelos traumas oculares graves, em indivíduos do sexo masculino, nas faixas etárias menor de vinte e um anos e maior de sessenta anos, devido aos materiais de construção.

O tipo de lesão ocular predominante foi a perfuração ocular, na sua grande maioria unilateral e responsável por um prognóstico visual reservado, apesar do tratamento cirúrgico.

Levando-se em consideração a elevada prevalência dos acidentes oculares graves em crianças, adolescentes e idosos do sexo masculino durante as suas atividades recreativas, é importante enfatizar a prevenção, orientando a população em geral e principalmente os responsáveis por tais indivíduos, tendo como principal alvo, os extremos das faixas etárias.
ABS TRACT

Purpose: To study the prevalence and circumstances of severe ocular trauma in a University Hospital of São José do Rio Preto - SP. Methods: A retrospective study was performed on 216 patients with severe ocular trauma who underwent surgery between September, 1986 to September, 2000. Sex, age, laterality, cause of trauma, type of trauma, activity during the accident and final vision after trauma were evaluated. Results: 216 patients of whom $173(80 \%)$ were male, were studied. The most frequent age was below 20 years in $94(43.5 \%)$ patients. The unilateral injuries predominated in $209(96.8 \%)$. The major activity during the accident was leisure in $88(40.7 \%)$ patients. The most frequent agent was construction materials in 82 (38\%) cases. Ocular perforation was the most frequent type of trauma in 84 (85.2\%) patients. Conclusions: Unilateral ocular perforations by construction materials predominated in male children, teenagers and old people during leisure.

Keywords: Eye injuries/etiology; Accidents occupational; Construction materials; Blindness/prevention \& control; Eye health

\section{REFERENCIAS}

1. Alves MR, José NK. O trauma ocular como causa de cegueira. Rev Med (São Paulo) 1997;76:297-302.

2. Moreira CA, Debert-Ribeiro M, Belfort R. Epidemiological study of eye injuries in Brazilian children. Arch Ophthalmol 1988;106:781-4.

3. Alves MR, José NK, Prado Junior J, Usuba FS, Onclinx TM, Marantes CR. Ferimento perfurante ocular: 400 casos admitidos na clínica oftalmológica do Hospital das Clínicas da Faculdade de Medicina da Universidade de São Paulo. Arq Bras Oftalmol 1995;58:342-5.

4. Bernucci EA, Lopreto RCC, Rodrigues MLV. Traumatismos oculares em uma unidade de emergência. Rev Bras Oftalmol 1993;52:407-11.

5. Carani JCE, Machado CG, Gomi CF, Carvalho RMS. Ferimentos perfurantes oculares no Hospital das Clínicas da Faculdade de Medicina da Universidade de São Paulo. O que mudou nos últimos 27 anos. Arq Bras Oftalmol 1999;62:310-4

6. Schellini SA, D’Aurea Filho JA, Padovani CR, Silva MRBM. Causas e características do trauma ocular perfurante em Botucatu - SP. Rev Bras Oftalmol 1995;54:829-34.

7. Fernandes S, Schwartz H, Cachuba PT, Sobreiro EM. Ferida perfurante do globo ocular. An Oftalmol 1991;10:41-4.

8. Bordon AF, Souza LB, Moraes NSB, Freitas D. Perfuração ocular: estudo de 473 casos. Arq Bras Oftalmol 1994;57:62-5.

9. José NK, Alves MR, Oliveira PR. Como educar a população para a prevenção do trauma ocular. Arq Bras Oftalmol 1992;55:160-2.

10. Schellini SA, Marchi NLM, Itoda LK, Silva MRBM, Sab N. Acidentes oculares graves decorrentes do trabalho. Rev Bras Oftalmol 1993;52:55-62.

11. Cohen J, Carvalho RC, Romão E. Trauma ocular por acidente de trabalho em Manaus (AM). Rev Bras Oftalmol 1994;53:149-52.

\section{Ao enviar um artigo para publicação, leia ATENTAMENTE as instruções para autores, constante no final de cada fascículo.}

